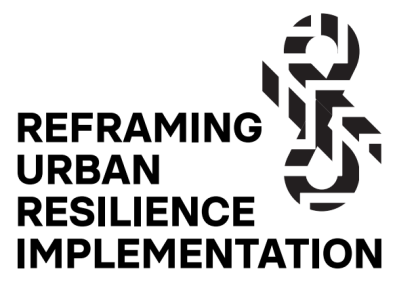

\title{
Social vulnerability and coastal hazards: Acknowledging floating population needs in Barcelona, Spain
}

\author{
Aysun Koroglu ${ }^{1 *}$, Lina Taing ${ }^{2}$ and Chris Zevenbergen ${ }^{1}$ \\ ${ }^{1}$ IHE Delft, Water Engineering Department, 2601 DA Delft, The Netherlands \\ ${ }^{2}$ IHE Delft, Environmental Engineering \& Water Technology Department, 2601 DA Delft, The \\ Netherlands
}

E-Mails: a.koroglu@un-ihe.org (A.K.); 1.taing@un-ihe.org (L.T.); c.zevenbergen@un-ihe.org (C.Z.)

* Author to whom correspondence should be addressed; Tel.: +31152151295 a.koroglu@un-ihe.org

\begin{abstract}
Increasing occurrences of flash flooding poses significant social and economic threats to Barcelona. Approximately $65 \%$ of the population reside along the coast. Many rely on beach assets to attract 35 million annual visitors that buttress the city's 7.1 billion EUR tourist sector. Both residents and tourists are vulnerable to late-summer and early-autumnal flash floods of intense rainfall events that that exceed the capacity of urban drainage systems designed for 55\% less loading. Government efforts do not account for non-resident population needs by focusing primarily on residents' safety following floods. Regular flash floods in Barcelona indicates an urgent need to develop a water sensitive strategy that comprehensively accounts for point source pollution in this vulnerable coastal region, as well as for its socioeconomic profile. While Social Vulnerability Indices have been developed for climate change-related disasters over the past fifteen years, these indices are designed for use at a national scale and overlook the needs of seasonal residents (e.g. short-term residents and tourists) in social profiling. This research broadens the scope of social vulnerability indices to factor in temporary resident needs in disaster planning at a regional scale. The social vulnerability index can help government planners include floating population groups in postdisaster management efforts.
\end{abstract}

Keywords: Social vulnerability index; SVI; resilience; seasonal population; disaster risk reduction. 


\section{Introduction}

Coastal metropolises are sensitive to hazards due to their having high concentrations of people and economic activities within their geographic boundaries (Guillard-Gonçalves et al., 2015). Previous climate change research has indicated that densely populated coastal areas are vulnerable to disasters such as flash floods (Velasco et al., 2018). Consequently, preparing for and minimizing vulnerability to hazards such as urban flooding is of interest to coastal cities. Social vulnerability (SV) is a population's inability to cope with adverse impacts of natural and/or man-made disasters. It encompasses characteristics that influence human susceptibility to disasters and their ability to respond, as well as context-specific social and environmental factors (such as urbanization and economic activities) (Cutter et al., 2003).

This case study focuses on the second most populous region in Spain (Barcelona). Approximately $65 \%$ of its population reside along the coast (idescat, 2018). Increasing occurrences of flash flooding pose significant socioeconomic threats to the province and its residents, as many rely on beach assets to attract 35 million annual visitors that buttress Barcelona's 7.1 billion EUR tourist sector (WTTC, 2017). Both residents and tourists are vulnerable to late-summer and early-autumnal flash floods of intense rainfall events that exceed the capacity of drainage systems designed for 55\% less loading (Santos and Martos, 2009).

This research follows in the tradition of SV exposure and resilience studies undertaken by Cutter, et al. (2003). As per previous research, we analyze available socioeconomic and demographic data to construct a Social Vulnerability Index (SVI) for Barcelona's coastal hazards to link the attributes that make people vulnerable to the support structures and facilities that can help them resist and recover (Gomes, 2018). We, however, diverge from Cutter, et al. (2003) in that we use municipal-level data instead of country-level data to develop an index that takes into account Barcelona's short-term residential and tourist population as vulnerable groups, as this floating population (població estacional) has thus far been overlooked in previous SVIs. Tourists especially are important to account for because Barcelona's tourist numbers increased by over 25\% between 2008-2015 (Barcelona City Council, 2015).

The study's aim is to inform the development of floating population variables. Developing means to assess this SV can facilitate our understanding of factors that make non-residents and places susceptible to disasters over time and in different places. It additionally can offer guidance to government officials responsible for prioritizing resources in emergency events (CDC, 2018) - especially in areas that are heavily reliant on tourism.

\section{Study Area \& methodology}

The study area covers coastal municipalities of Barcelona Province (41N, 2.1E UTM coordinates). The coastline is approximately 160 kilometres in length and is relatively narrow with a high elevation. It comprises of 103 beaches distributed along four coastal administrative units (comarcas: Maresme, Barcelonès, Llobregat and Garraf) (Figure 1). According to idescat (2018), 5.5 million people reside in these coastal comarcas. 
Figure 1. (a) Study area map

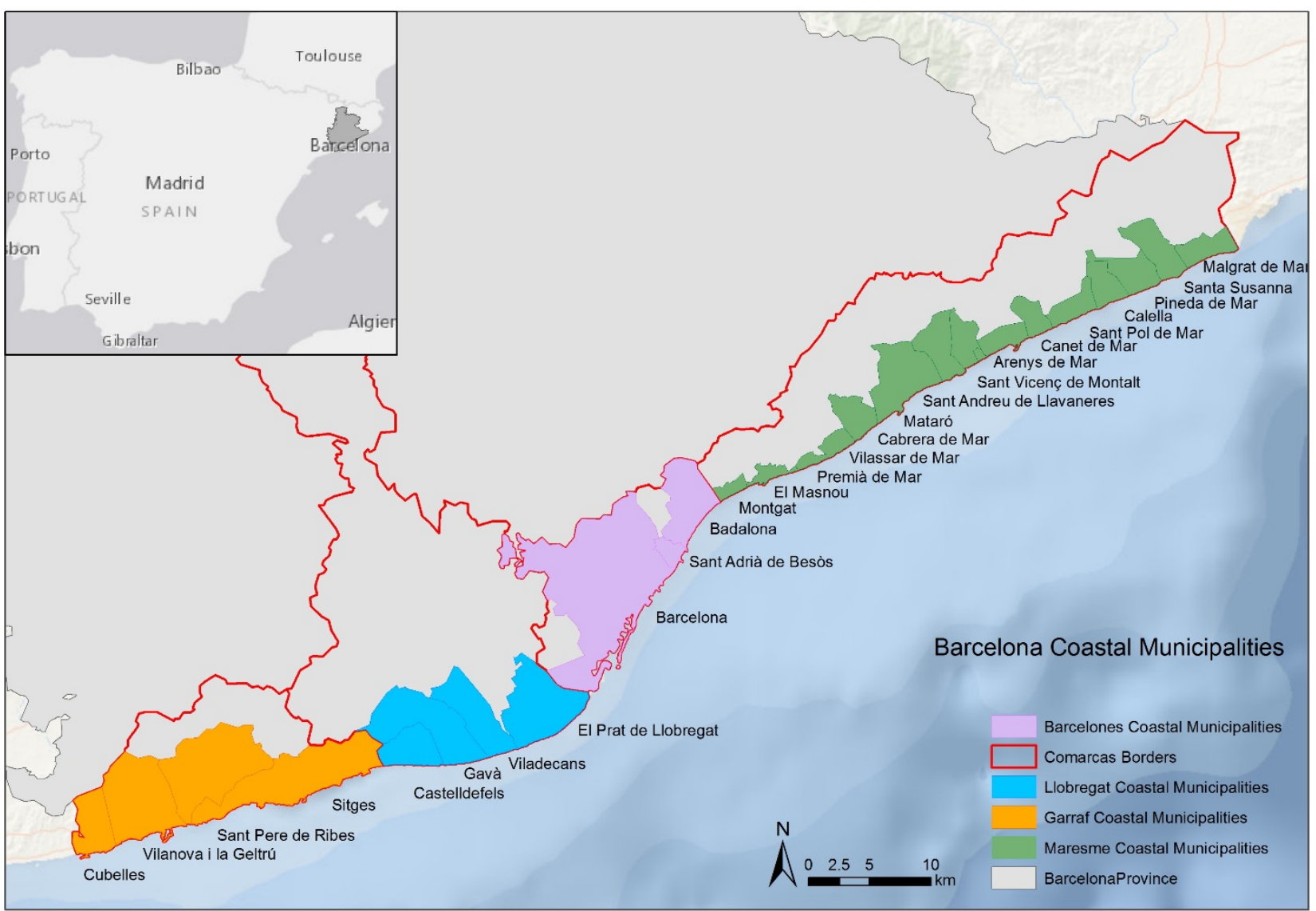

(b) Created in Arc GIS 10.5.1

We examined both residential and floating populations' SV to coastal hazards in 25 municipalities located within these four comarcas. (We excluded a further 3 municipalities with $<5,000$ inhabitants due to their having unreliable data.) Data is limited to the 25 municipalities due to the study's coastal hazards objective. In this study, we applied the US Federal Emergency Management Agency's SV tool using R scripts to prepare and analyze data (FEMAdata, 2016; R Development Core Team, 2008).

\subsection{Data collection and preparation}

Table 1 summarizes the variables used for analysis. The first 28 variables are based on SVI research from Cutter et al. (2003) and Guillard-Goncalves et al. (2014) and Martin et al.'s (2017) adaptations for European contexts. Variables development accounted for European contexts - such as foreign nationals living in Spain, as Guillard-Goncalves et al. (2014) noted that ethnicity components are not included in the Cutter framework. The latter 5 floating population variables are drawn from Barcelona's latest municipal (2011) census (idescat, 2018). Barcelona's statistics institution (idescat) includes in its floating population those who have a primary or secondary municipal residence; tourists who stay overnight at tourism establishments; and students or workers who travel between municipalities but do not stay overnight. Idescat (2016) bases floating population figures on censuses and tourism, labor, education and lifestyle statistics. We grouped the 33 variables in 11 categories to reflect socioeconomic attributes or support structures: housing and installations, age, race and ethnicity, education, family structure, urban, socioeconomic status, medical services, special needs population, population growth, and floating population. 
Table 1. Variables names and descriptions

\begin{tabular}{|c|c|}
\hline Code Name & Description \\
\hline \multicolumn{2}{|l|}{ Housing and services } \\
\hline PriWatSup & Proportion of dwellings with private water supply/Total dwellings \\
\hline NoWatSup & Proportion of dwellings with no water Supply/Total dwellings \\
\hline NoHeat & Proportion of dwellings with no heating/Total dwellings \\
\hline NoPhone & Proportion of dwellings with no telephone line/Total dwellings \\
\hline NoBath & $\begin{array}{l}\text { Proportion of dwellings with no toilet, bathroom or shower inside the } \\
\text { apartment/Total dwellings }\end{array}$ \\
\hline NoCool & Proportion of dwellings with no cooling/Total dwellings \\
\hline \multicolumn{2}{|l|}{ Age } \\
\hline Elderly & Population 65 and older/ Population 15-64 years \\
\hline Young & Population under 15 / Population 15-64 year \\
\hline \multicolumn{2}{|l|}{ Race and ethnicity } \\
\hline Spanish & Proportion of population with Spanish Nationality/Total Resident Population \\
\hline Foreign & Proportion of population with Foreign Nationality/Total Resident Population \\
\hline \multicolumn{2}{|l|}{ Education } \\
\hline Element & Proportion of people elementary school graduates/Total number of graduates \\
\hline Univer & Proportion of population who completed College/Total number of graduations \\
\hline \multicolumn{2}{|l|}{ Familial structure } \\
\hline MothersAlone & Proportion of mothers alone with child/total number of families \\
\hline ParentsAlone & Proportion of parents alone with child/total number of families \\
\hline HouseholdSize & Average number of people per household \\
\hline \multicolumn{2}{|l|}{ Urban } \\
\hline Density & Number of people/area (in $\mathrm{km}^{2}$ ) \\
\hline \multicolumn{2}{|c|}{ Socioeconomic status } \\
\hline GDP & Income per capita (thousands of Euros) \\
\hline RatUnemLabor & Ratio of unemployed population and labor force \\
\hline Act.Rate & Rate of activity \\
\hline Agri & Proportion of population employed in agriculture/Active population \\
\hline Tou & Proportion of population employed in tourism/Active population \\
\hline Renters & Proportion of rented or sub-rented conventional dwellings/Total Dwellings \\
\hline \multicolumn{2}{|l|}{ Medical services } \\
\hline Phar & Number of pharmacies for 1000 inhabitants (in 2002) \\
\hline HosBeds & Number of hospital beds for 1000 inhabitants (in 2002) \\
\hline \multicolumn{2}{|c|}{ Special needs population } \\
\hline Disabled & Proportion of disabled persons (auditory, visual, motor or mental) \\
\hline DisabledDep & Proportion of persons that are disabled and are under 4 or above 65 years old \\
\hline Disabl60 & Proportion of persons with a disability degree above $60 \%$ \\
\hline \multicolumn{2}{|l|}{ Population growth } \\
\hline NetMigra & Net Migration (annual average) (rate per 1.000 inhabitants). 2001-2011 \\
\hline \multicolumn{2}{|l|}{ Floating population } \\
\hline BedHotCamp & Number of beds in hotels \& camping \\
\hline NonResPop & Non-resident population \\
\hline TouEst & Estimated number of tourists results for the period 2002-2015 \\
\hline RatPopVicandRes & Floating estimates for the period 2002-2015/ resident population \\
\hline nonres_Res & Non Resident Population_Tourist/Resident Population \\
\hline
\end{tabular}


To prepare data, we applied a multi-collinearity (Kendall's rank correlation) test to reduce data amplitude because all variables do not have normal distributions. We discarded the Disabl60 variable from the dataset because the Kendall s-b value was below the accepted correlation rate of \pm 0.7 .

\subsection{PCA with varimax rotation}

This is performed to select key principal components (PCs), which facilitates data interpretation. We (1) applied a varimax rotation procedure using the Kaiser Criterion; and (2) normalized the variables with Z-scores. For variables with an eigenvalue higher than one, we excluded those with correlation coefficients $<0.4$ as they likely do not contribute to $\mathrm{SV}$. We examined the remaining variables by assigning a positive or negative contribution to vulnerability, and considered how socially vulnerable each municipality in the dataset is to coastal hazards.

\subsection{SVI calculation \& GIS visualization}

We mapped equally weighted SVI scores in ArcGIS 5.1 using standard deviation classifications of very high to very low vulnerability classes (Figure 2). To note, as per previous SVI frameworks, a positive sign indicates increased vulnerability and a negative sign indicates a diminishing effect (Table 2). We used an absolute value symbol when the variables within the category conflict in contribution. If a component's variables tend to increase SV but have a negative loading (or vice-versa), the component's cardinality is adjusted by multiplying by -1 . A SVI is calculated by summing the component values for each municipality (Equation 1).

$$
\text { SVI Score }=\mathrm{PC}_{1}+\mathrm{PC}_{2}+\mathrm{PC}_{3}+\mathrm{PC}_{4}+\left|\mathrm{PC}_{5}\right|-\mathrm{PC}_{6}+\mathrm{PC}_{7}-\mathrm{PC}_{8}+\mathrm{PC}_{9}+\mathrm{PC}_{10}
$$

\section{Results}

Twenty variables remained after processing, which were clustered according to ten PCs with 87.3\% variance. However, we found that three variable combinations (PC3: NoHeat and Disabled; PC7: NoBath and NoCool; PC9: NoWatSup and Agri) have limited relevance to SV in coastal hazard events, and hence excluded these PCs. The variance for the remaining PCs is $66.7 \%$ (Table 2).

Table 2. SV components, attributed signs and correlation

\begin{tabular}{|c|c|c|c|c|c|}
\hline PC & Variance $(\%)$ & Vulnerability categories & Sign & Variables & Correlation \\
\hline \multirow{3}{*}{1} & \multirow{3}{*}{26.2} & \multirow{3}{*}{ Floating population } & \multirow{3}{*}{+} & BedHotCamp & 0.439 \\
\hline & & & & NonResPop & 0.449 \\
\hline & & & & TouEst & 0.428 \\
\hline \multirow{3}{*}{2} & \multirow{3}{*}{12.6} & \multirow{3}{*}{$\begin{array}{l}\text { Housing services \& } \\
\text { population growth }\end{array}$} & \multirow{3}{*}{+} & PriWatSup & 0.559 \\
\hline & & & & NoPhone & -0.411 \\
\hline & & & & NetMigra & -0.403 \\
\hline \multirow{2}{*}{4} & \multirow{2}{*}{9.3} & \multirow{2}{*}{$\begin{array}{l}\text { Education \& socioecon. } \\
\text { status }\end{array}$} & \multirow{2}{*}{+} & Element & -0.468 \\
\hline & & & & RatUnemLabor & -0.486 \\
\hline \multirow{2}{*}{5} & \multirow{2}{*}{5.9} & \multirow{2}{*}{ Race \& ethnicity } & \multirow{2}{*}{ | } & Spanish & -0.521 \\
\hline & & & & Foreign & 0.472 \\
\hline 6 & 5.4 & Medical services & - & Phar & -0.463 \\
\hline
\end{tabular}




\begin{tabular}{|c|c|c|c|c|c|}
\hline $\mathbf{8}$ & 4.1 & Socioeconomic status & - & GDP & -0.567 \\
\hline $\mathbf{1 0}$ & 3.2 & Age \& Familial structure & + & Young & -0.547 \\
\hline
\end{tabular}

After identifying relevant PCs, we mapped the municipalities' SV according to standard deviation distributions of very high $(>1.5)$ to high $(1.5$ to 0.5$)$, moderate $(0.5$ to -0.5$)$ or low $(<-0.5)$. Taking into account the SVI score, the most vulnerable municipalities are Barcelona City and Cubelles, while Vilanova i la Geltru, Sitges, Castelldefels, Gava, Teia, Vilassar de Mar, Mataro, Canet de Mar and Pineda de Mar are the least vulnerable (Fig. 2).

Figure 2. (a) SV spatial distribution categories in coastal municipalities of Barcelona Province.

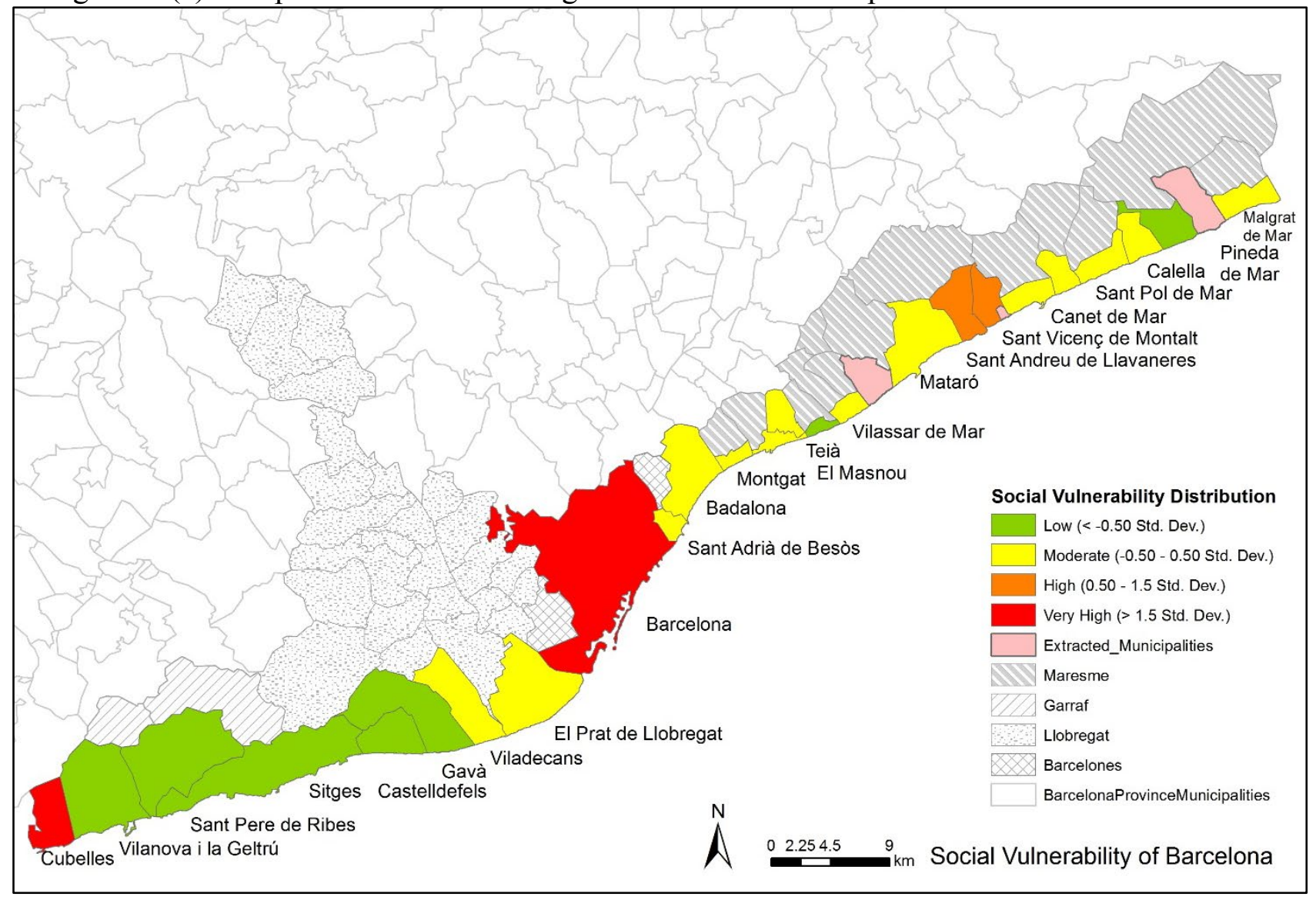

(b) Created in Arc GIS 10.5.1

Both education and socioeconomic status contribute to SV in Barcelona City and Cubelles. Barcelona City seemed to also very vulnerable due to its floating population, whereas wealth was the second contributory factor that affected Cubelles' classification. The nine least vulnerable municipalities did not have any very high PC scores. They additionally each have at least three moderate PC scores, with an overall average score of moderate. Housing services and population growth (PC2) contribute to SV in four of the nine municipalities, whereas sufficient medical services (PC6), wealth (PC8) and/or age/familial structure (PC10) seemed to lower vulnerability.

In terms of the primary contributory factor, the SVI scores indicate that floating populations (PC1) has the highest variance, which suggests that this cluster contributes most amongst the 25 coastal municipalities. Barcelona City is most vulnerable, though it is also a high contributor in four other municipalities (Badolona, Arenys de Mar, Calella and Pineda de Mar). Floating populations are a low 
contributor in eight municipalities (Cubelles, Sant Pere de Ribes, Viladecans, Teia, Mataro, Sand Andreu de Llavaneres, Sant Vicenc de Montalt and Sant Pol de Mar).

\section{Conclusion}

This study evaluated the SV of coastal municipalities in Barcelona Province against hazards such as flash floods. We applied the SVI framework to assess which are the most socially vulnerable populations in 25 coastal municipalities. We found that floating populations are the most vulnerable to coastal hazards. This is important to highlight to decision-makers responsible for government disaster risk reduction programs, as this suggests that emergency services should also account for short-term residential and tourist population needs in areas where tourism is a significant economic sector. Using SVI maps can additionally inform how finances and services can be spatially distributed according to risks.

This study joins a body of research that indicates that the SVI framework can inform disaster risk reduction decision-making and prioritization. The findings are also important because the SVI framework presently does not account for non-residential populations in its analysis. This paper thus serves as an opportunity to inform the development of a critical but overlooked variable for contexts that experience high rates of short-term residential and/or tourist populations.

Due to time and resource limitations, we limited the dataset to 25 Barcelona municipalities. We plan to expand this analysis across coastal municipalities in Catalonia to build a more statistically robust index for this region. And while determining and mapping SV factors have the potential to improve disaster response processes, its wider adoption is dependent on there being reliable government data and public institutions having basic statistical analysis knowledge in order to integrate and map statistical and spatial data.

\section{Acknowledgments}

We appreciate the statistical assistance that Yago Martin Gonzalez provided in the drafting of this paper. The first two authors received funding from the European Union's Seventh Framework Programme for research, technological development and demonstration under grant agreement no 606838, with additional support from IHE Delft, to conduct this research.

\section{Conflict of Interest}

The authors declare no conflict of interest.

(C) 2018 by the authors; licensee MDPI and IFoU, This article is an open access article distributed under the terms and conditions of the Creative Commons Attribution license. 